\title{
Capsule Commentary on Dahlke et al., The Mini Mental Status Exam as a Surrogate Measure of Health Literacy
}

\author{
Michael H. Perskin, MD \\ Divisions of GIM \& Geriatrics, Department of Medicine, NYU School of Medicine, New York, NY, USA.
}

J Gen Intern Med 29(4):650

DOI: $10.1007 / \mathrm{s} 11606-013-2739-z$

(c) Society of General Internal Medicine 2014

$\mathrm{L}^{\mathrm{o}}$ ow health literacy is a common problem in primary care that has implications for health and health outcomes. ${ }^{1,2}$ Dahlke et al. ${ }^{3}$ compared performance on the Mini Mental Status Exam (MMSE) with three common health literacy instruments. The data demonstrated weak correlations (ranging from 0.52 to 0.70 ) between all three literacy instruments and the MMSE, with scores less than 27 predicting impaired health literacy. The authors conclude that the MMSE and health literacy instruments may be measuring similar latent constructs.

It may not be surprising that worse cognition is correlated with lower literacy. Literacy is a cognitive construct. What is surprising is that this relationship was found at MMSE cut points that are considered relatively normal. Not all components of the MMSE were equally correlated with diminished literacy. Serial subtraction and praxis were the most highly correlated components. Given that numeracy and literacy are often similarly diminished, it may not be surprising that serial subtraction on the MMSE had one of the stronger correlations with literacy. Praxis, difficulty with drawing, is an indication of frontotemporal problems, the area of the brain involved in reading.

The authors suggest that the MMSE may be used as a proxy for identifying diminished literacy. Since the MMSE is no longer freely available, its use for measuring literacy may not be tenable, given the availability of public domain literacy instruments. Clinically, rather than using diminished MMSE scores as a proxy for literacy, perhaps patients with diminished literacy should be evaluated for impaired cognition. It seems likely that literacy and cognition measure similar latent constructs, just as trust and satisfaction are often tightly related. While trust and satisfaction are often highly correlated and have overlapping factors that underlie the meaning of each construct, to patients the differences between the two are real, though subtle. Further research on literacy, numeracy and their relationship with cognition is merited.

Conflict of Interest: The author has no conflicts of interest with this article.

Corresponding Author: Michael H. Perskin, MD; Divisions of GIM \& Geriatics, Department of Medicine, NYU School of Medicine, New York, NY, USA (e-mail: Michael.perskin@nyumc.org).

\section{REFERENCES}

1. Baker Dw. Reading between the lines: deciphering the connections between literacy and health. J Gen Intern Med. 1999;14(5):315-7.

2. Hasnain-Wynia R, Wolf MS. Promoting Health Care Equity: Is Health Literacy a Missing Link? Health Serv Res. 2010;45(4):897-903.

3. Dahlke, AR, Curtis LM, Federman AD, Wolf MS. The Mini Mental Status Exam as a Surrogate Measure of Health Literacy. J Gen Intern Med. [SPI: 2712]. 